\title{
Rational Chebyshev Approximations for the Error Function*
}

\author{
By W. J. Cody
}

\begin{abstract}
This note presents nearly-best rational approximations for the functions erf $(x)$ and erfc $(x)$, with maximal relative errors ranging down to between $6 \times 10^{-19}$ and $3 \times 10^{-20}$.
\end{abstract}

In [1] Hart, et al., present rational approximations for the function

$$
\operatorname{erfc}(x) \equiv 1-\operatorname{erf}(x)=\frac{2}{\sqrt{ } \pi} \int_{x}^{\infty} e^{-t^{2}} d t
$$

valid for $0 \leqq x \leqq \alpha$, where $\alpha=4,8,10$, or 20 . They carefully point out [1, p. 138] that these approximations are not useful for computing the error function

$$
\operatorname{erf}(x) \equiv 1-\operatorname{erfc}(x)=\frac{2}{\sqrt{ } \pi} \int_{0}^{x} e^{-t^{2}} d t
$$

for small $x$ because of subtraction error, but they do not provide any alternative. Hastings' [2] approximations for erf $(x)$ are no better, since they explicitly use the constant 1 as an additive term and are chosen to nearly minimize the maximum absolute error rather than the relative error. Clenshaw's [3] Chebyshev series expansions for erf $(x) / x$ come close to minimizing relative error, but his approximations are somewhat inefficient because of his choice of interval and his restriction to polynomials.

For a computer subroutine with entries for both erf $(x)$ and erfe $(x)$, cancellation error can be avoided by evaluating erf $(x)$ directly and erfe $(x)$ indirectly (as 1 - erf $(x))$ when erf $(x)$ is smaller in magnitude than erfe $(x)$, and erf $(x)$ indirectly and erfe $(x)$ directly, otherwise. The changeover point occurs for $|x| \simeq .47$.

In this note we present nearly-best rational approximations for the functions erf $(x)$ and erfe $(x)$ with maximal relative errors ranging down to between $6 \times 10^{-19}$ and $3 \times 10^{-20}$. The approximation forms and intervals used are

$$
\begin{aligned}
\operatorname{erf}(x) & \simeq x R_{l m}\left(x^{2}\right), \quad|x| \leqq .5 \\
\operatorname{erfc}(x) & \simeq e^{-x^{2}} R_{l m}(x), \quad .46875 \leqq x \leqq 4.0, \\
\operatorname{erfc}(x) & \simeq \frac{e^{-x^{2}}}{x}\left\{\frac{1}{\sqrt{ } \pi}+\frac{1}{x^{2}} R_{l m}\left(1 / x^{2}\right)\right\}, \quad x \geqq 4,
\end{aligned}
$$

where the $R_{l m}(z)$ are rational functions of degree $l$ in the numerator and $m$ in the denominator. The relations erf $(-x)=-\operatorname{erf}(x)$ and erfc $(-x)=2-\operatorname{erfc}(x)$ can be used to evaluate the functions for negative arguments.

Received January 24, 1969).

* Work performed under the auspices of the U. s. Atomic Energy Commission. 
Table I. $\varepsilon_{l m}=-100 \log _{10} \max \left|\frac{f(x)-f_{\ell m}(x)}{f(x)}\right|$

$$
f(x)=\operatorname{erf}(x), \quad|x| \leq .5
$$

$f^{* * * * * * * * * * * * * * * * * * * * * * * * * * * * * * * * * * * * * * * * * * * * * * * * * * * * * * * * *}$

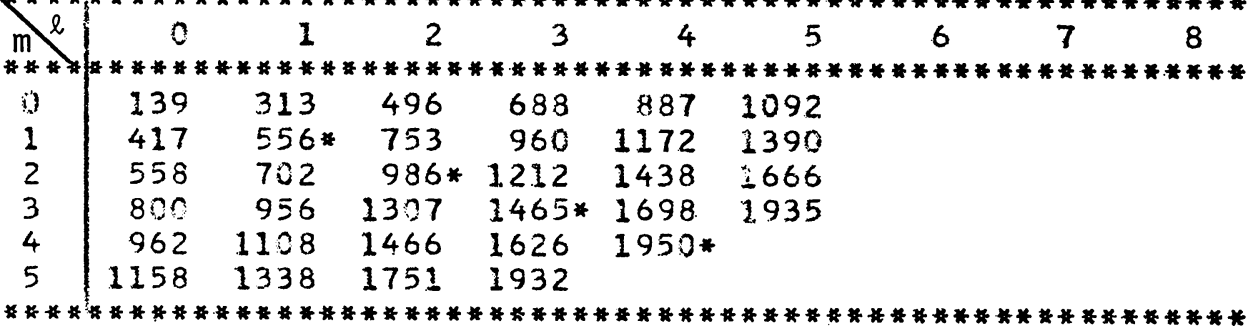

$f(x)=\operatorname{erfc}(x), \quad .46875 \leq x \leq 4.0$

\begin{tabular}{|c|c|c|c|c|c|c|c|c|c|}
\hline $\begin{array}{l}0 \\
1 \\
2 \\
3 \\
4 \\
5 \\
6 \\
7 \\
8\end{array}$ & 164 & $\begin{array}{c}61 \\
222 \\
376 \\
440 \\
502\end{array}$ & $\begin{array}{l}109 \\
280 \\
441 \\
597 \\
666\end{array}$ & $\begin{array}{l}161 \\
340 \\
506 \\
666 \\
824\end{array}$ & $\begin{array}{c}21.4 \\
401 \\
572 \\
736 \\
897 * \\
1056\end{array}$ & $\begin{array}{c}270 \\
462 \\
638 \\
806 \\
1132 * \\
1292\end{array}$ & $\begin{array}{l}1371 \\
1532\end{array}$ & $\begin{array}{l}1613 * \\
1775\end{array}$ & $1859 *$ \\
\hline
\end{tabular}

$$
f(x)=\operatorname{erfc}(x), \quad x \geq 4.0
$$

\begin{tabular}{|c|c|c|c|c|c|}
\hline $\begin{array}{l}0 \\
1 \\
2 \\
3 \\
4 \\
5\end{array}$ & $\begin{array}{c}688 * \\
855 \\
992 \\
1116 \\
1232\end{array}$ & $\begin{array}{c}628 \\
828 \\
998 * \\
1151 \\
1283 \\
1405\end{array}$ & $\begin{array}{l}756 \\
958 \\
1131 \\
1287 * \\
1431 \\
1558\end{array}$ & $\begin{array}{r}876 \\
1081 \\
1256 \\
1415 \\
1561 *\end{array}$ & 1824* \\
\hline
\end{tabular}

*Coefficients for these approximations only are given in Tables II-IV. 


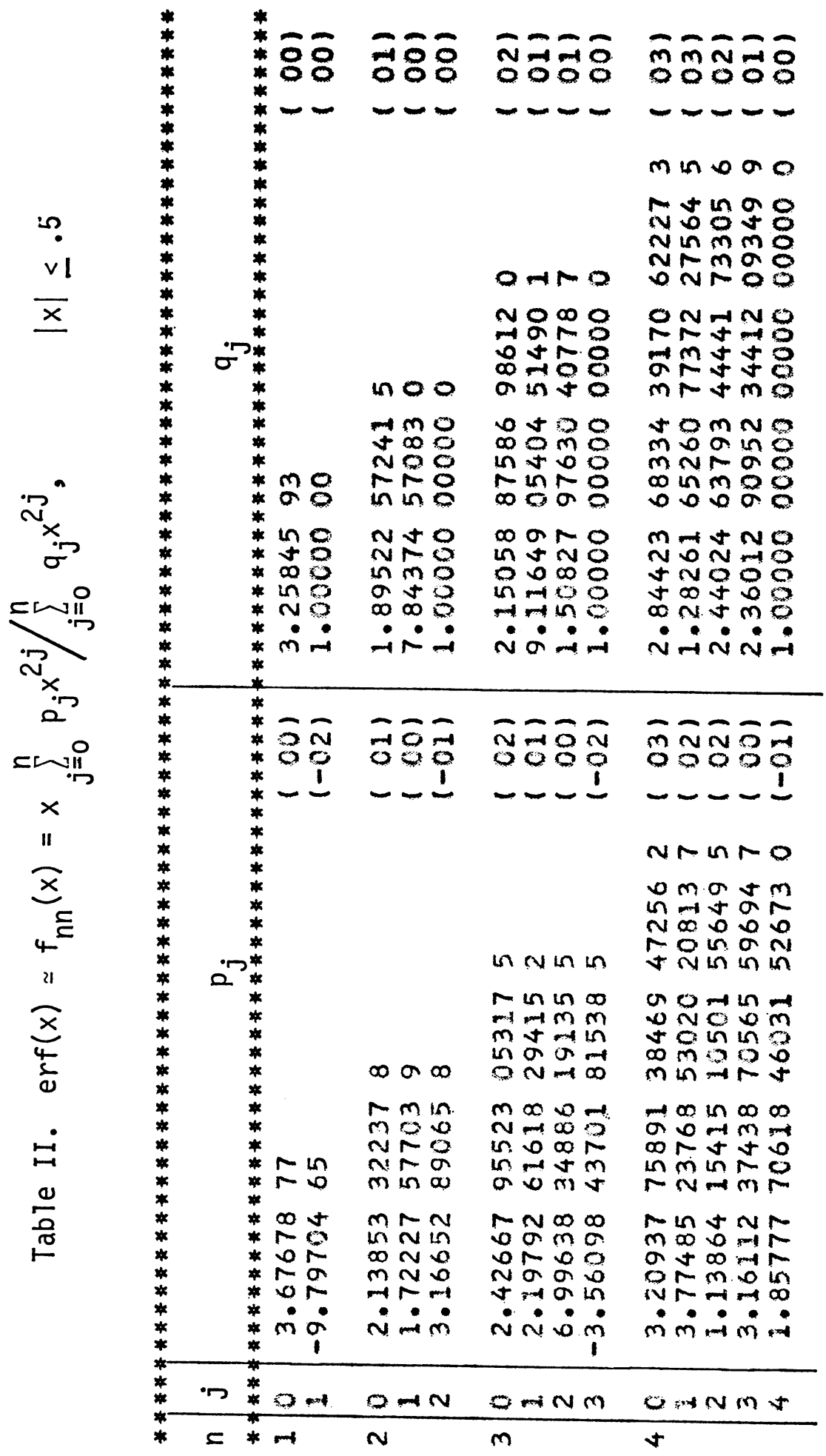




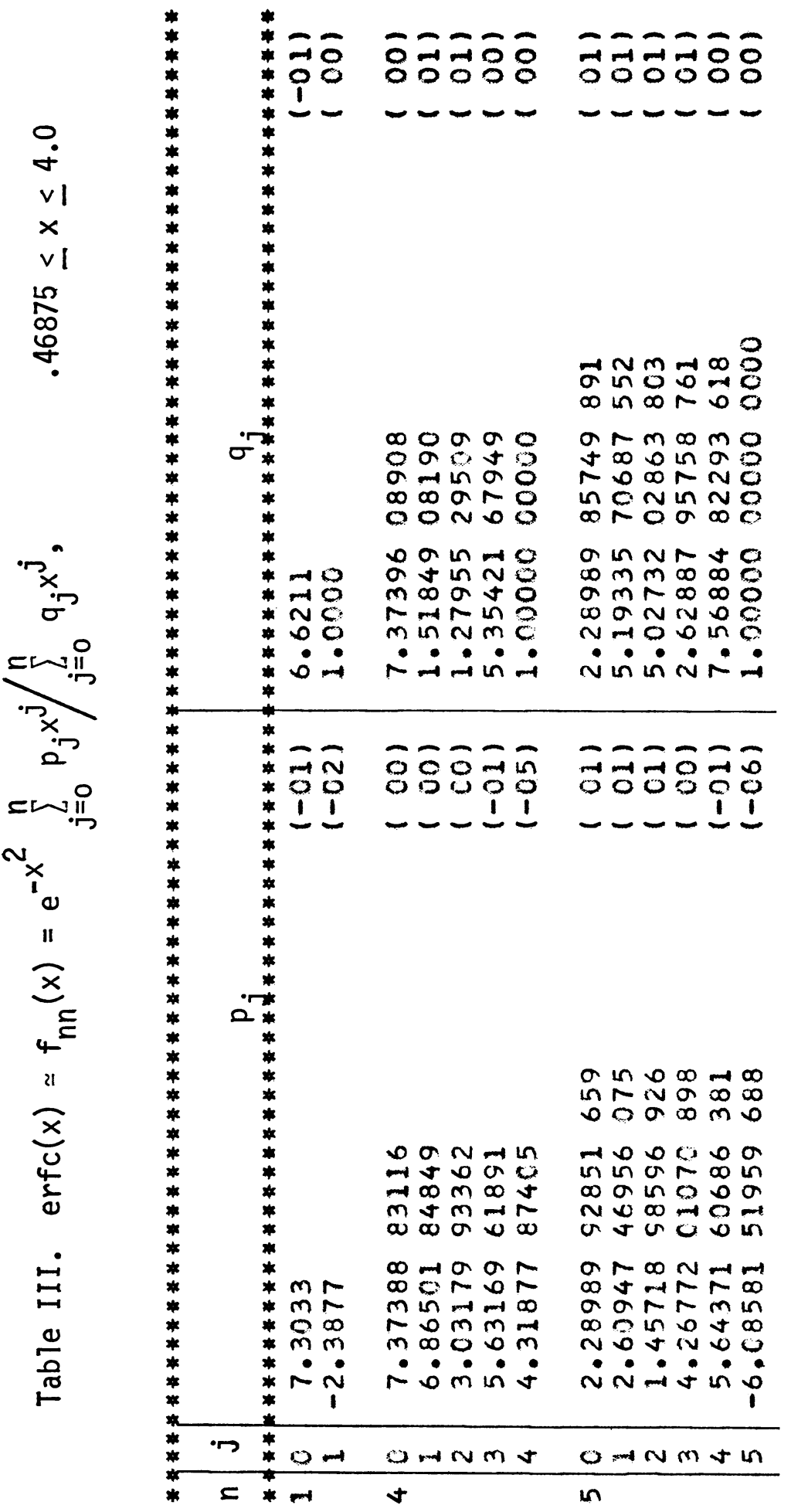


ลักิกักิกัละ์

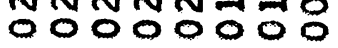

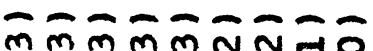

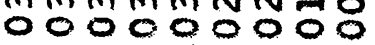

$m N=i n+i n m 0$ $m \sim m \infty m a$ in 0 a N N 0 I N

$m \sim 0 \infty+0$ in $m ?$ $00 \infty$ in $m$ in

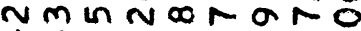
$+0+0=$ in $\alpha+0$

NoOm ONNO $m \infty a+r+40$ $\infty \sigma 0 \mathrm{~m} \infty \mathrm{a} \sigma 0$ a $\infty$ b o 0 N O $\mathrm{N}$ in $\mathrm{m} N 0$

a m $\operatorname{ros} 00 \mathrm{t} 0$

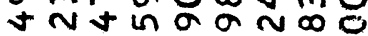
NNN $m m m m b 0 m 00$ $0+\operatorname{moN}$ N

in $N$ in $0 \pm$ in 00 a $m \infty \pm N m-\infty$ $\theta$ in $t+a m o$ ONa otNRO NaON

$\infty \mathrm{mm}$ - I 0000 $\pi+\infty$ in $t \infty \infty \infty+\infty 0$ UNamN-m, moON in 0 in 00 a $a \sigma a m a n=$

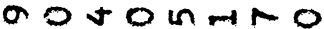
in in $\operatorname{co} \infty \mathrm{N} 0$ tama in 0 Do $00-\infty N \infty N$

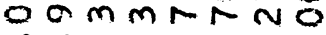
in: $: 0$. $m \infty-a \infty m m a$ $m m$ or $m \infty \propto ⿻$ o 2 Nom- ovo $m m \circ \sigma N R N R O$ $N+m \sim \circ m m$ L - $\bullet \cdot . \bullet$ $m \sim a 0 N N=$

$\rightarrow m \leqslant m-n \rightarrow-1$

जกतก $00 \mathrm{~N}$ 年 $-\ldots-2-1$

m̄ m 0000000 $--\infty---21$

in NOOOOMN rNAm min -0 $\Rightarrow+\infty 0$ in 0

o o o $\mathrm{rmm}$ ar - Nmoromo DN 5 t 0 ON , $\infty \mathrm{ma}$ in $m$ a $N$ $0 m \leqslant$. 000

$N+m+N \infty \sim \infty$ ONRONONm - $m$ o in $N$ in $N$

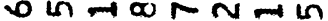
(von $\infty$ UN $\infty$ is

a 0 o 9 nin uns in $N \infty N N \sigma 0$ * a $m$ a 10 - $\infty$ $0-a N-m+6$ $O$ in. $m$ in $m N$ v - $\bullet \cdot ! \cdot$ $m+m-5 N$.

1
$N N v-N$ N $\infty=m$ $N m-m \sim m+\infty$ $N$ in $m+m R-m$ n $0 \propto 0-15 t a 0$

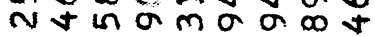

N-MOMNU1000 aN $N$ a O O S M $00000 m m \wedge 0$

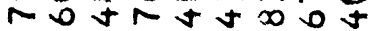
anm $\rightarrow N \rightarrow \infty \infty$

$\sim \infty \infty+\sigma N m \infty N$ TNNMm $\mathrm{N} \sigma \mathrm{v}$ in $N \rightarrow-\infty$ o 0 \% $m m$ onmoram or a N o on in

$m \sim \pm N$ In $-\infty+\infty$ $m o \sigma$ in $m a-\infty=$ $0 \cdots \sim \mathrm{N} 0 \mathrm{~m}-\mathrm{m}$ $m$ in $\rightarrow+\infty-\infty \pm$ in NUR - $\bullet \bullet \bullet \bullet$.

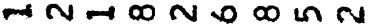

\begin{tabular}{|c|c|}
\hline $0-N m+n N M$ & $0 m N m+$ in $0 N \infty$ \\
\hline$N$ & $\infty$ \\
\hline
\end{tabular}




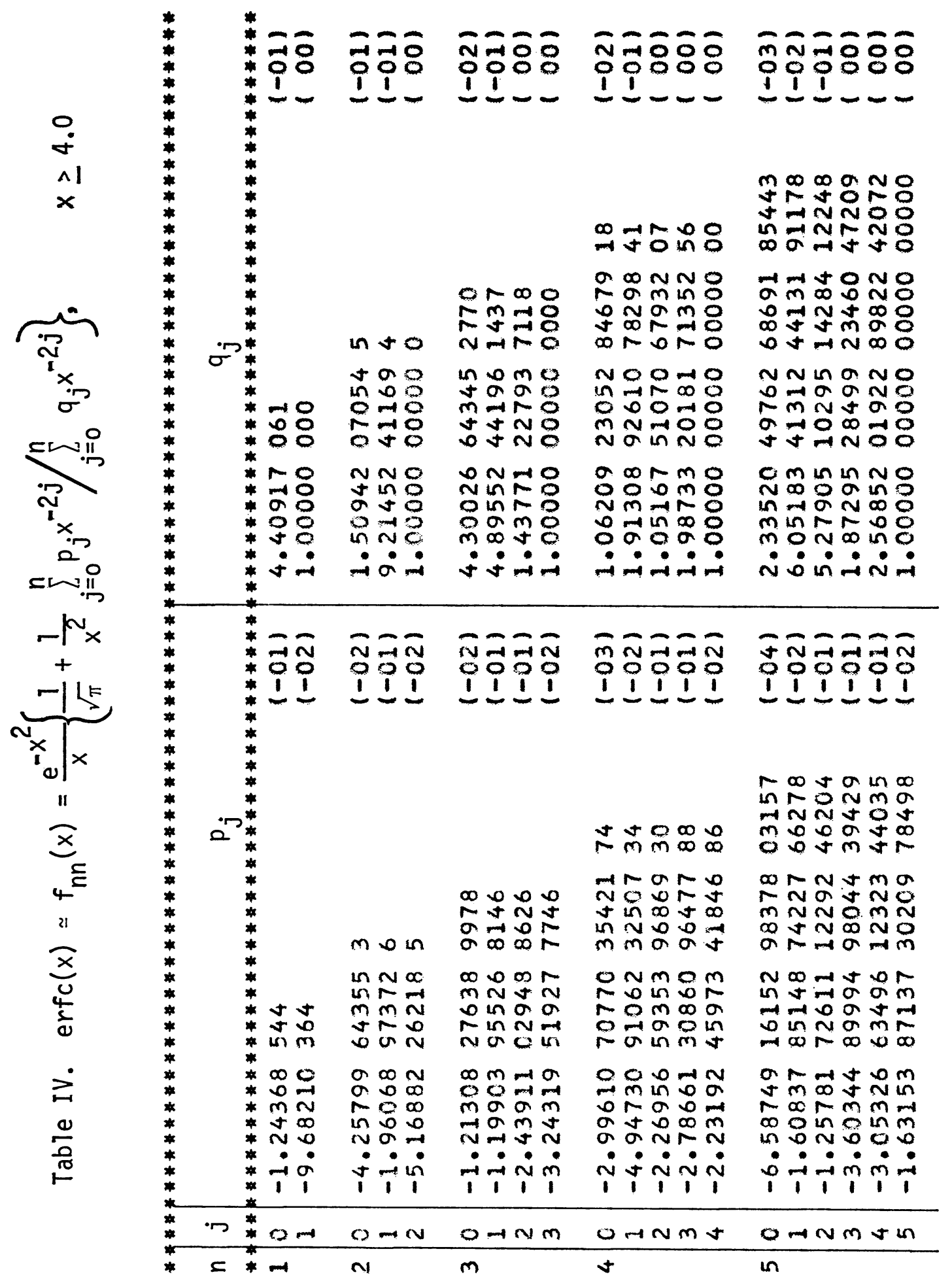


Table I presents the initial segments of the $L_{\infty}$ Walsh arrays while Tables II, III, and IV present selected approximations. All approximations were generated using a standard version of the Remes algorithm [4] on a CDC 3600. The master function routines used continued-fraction expansions described in [1] and were verified to be accurate to at least $22 \mathrm{~S}$. Finally, the accuracy of the approximations as presented here was verified by comparison against the master routines using 5000 pseudorandom arguments.

Argonne National Laboratory

Argonne, Illinois 60439

1. J. F. HART, et al., Computer Approximations, Siam Series in Appl. Math., Wiley, New York, 1968.

2. C. HAstings, JR., Approximations for Digital Computers, Princeton Univ. Press, Princeton, N. J., 1955. MR 16, 963.

3. C. W. Clenshaw, Chebyshev Series for Mathematical Functions, National Physical Lab. Math. Tables, vol. 5, H.M.S.O., London, 1962. MR 26 \#362.

4. W. J. CODY, W. Fraser \& J. F. HART, "Rational Chebyshev approximations using linear equations," Numer. Math., v. 12, 1968, pp. 242-251. 